\title{
Detection of Cryptosporidium sp. Oocyst and Giardia sp. Cyst in Faucet Water Samples from Cattle and Goat Farms in Taiwan
}

\author{
Yuko WATANABE ${ }^{1)}$, Kenji KIMURA ${ }^{2)}$, Cheng-Hsiung YANG $^{3)}$ and Hong-Kean OOI ${ }^{1) *}$ \\ ${ }^{1)}$ Department of Veterinary Medicine, National Chung Hsing University, 250 Kuo Kuang Road, Taichung 40227, Taiwan, ${ }^{2)}$ Water Quality \\ Central Laboratory, Maezawa Industries Inc., 5-11, Nakamachi Kawaguchi, Saitama 332-8556, Japan and ${ }^{3)}$ Livestock Disease Control \\ Center of Taichung County Taiwan, 21-1, Si-An Street, Feng-Yuan 42010, Taiwan
}

(Received 23 February 2005/Accepted 10 August 2005)

\begin{abstract}
A survey on the presence of Cryptosporidium oocyst and Giardia cyst in livestock drinking water as well as the urban tap water throughout Taiwan was carried out. Water examination for the presence of the protozoa was conducted by filtering through a PTFE membrane followed by immunomagnetic separation (IMS) and immunostaining the sediment with commercially available monoclonal antibody against Cryptosporidium and Giardia. Of the 55 different water samples from various sources examined, 2 were found to contain both of Cryptosporidium oocyst and Giardia cyst, 1 was found to contain Cryptosporidium oocyst only. These protozoa-positive water samples, originating from underground well and from the mountain spring, were also used as drinking water for livestock. However, no Cryptosporidium oocyst was found in the city tap water. This is the first report of Cryptosporidium oocyst and Giardia cyst being found in the drinking water for livestock.
\end{abstract}

KEY WORDS: Cryptosporidium, Giardia, livestock drinking water.

Cryptosporidium spp. and Giardia spp. cause diarrhea in a wide range of vertebrates including humans. This is particularly serious in neonatals and immunosuppressed individuals [1]. In the past decade, several waterborne outbreaks of human cryptosporidiosis $[10,14]$ suspected to be linked to water sources contaminated by Cryptosporidium oocysts derived from infected animals in farms had been reported $[11,14]$.

Cryptosporidium oocysts and Giardia cysts have been reportedly detected in river water in many countries $[4,13$, 15], including Taiwan [5]. However, the presence of Cryptosporidium or Giardia in livestock drinking water or treated tap water in city in Taiwan has not yet been investigated. An outbreak of cryptosporidiosis among goat kids in Taitung [12], and the presence of C. parvum oocysts in cattle feces in Taiwan had been reported [19]. Moreover, Cryptosporidium and Giardia had been reported in raw waters from the hog-farming region [5]. Thus, Cryptosporidium infection in livestock caused by drinking such parasites contaminated water might be a potential source of human cryptosporidiosis outbreaks in Taiwan.

In recent years, many farms designed for recreational purposes have been set up in Taiwan. This has provided ample opportunities for the public to be in close contact with farm animals, especially goat and cattle. Thus, there is an urgent need to examine whether the water used in the dairy farm, for both general use and as drinking water for the cattle, are contaminated with Cryptosporidium and Giardia or not. If these parasites are present in drinking water for livestock, the opportunity of contact with contaminated water by people coming to the farms for recreation or for work may be

\footnotetext{
* Correspondence to: Dr. OoI, H.-K., Department of Veterinary Medicine, National Chung Hsing University 250 Kuo Kuang Road, Taichung 40227, TAIWAN.
}

increased. Moreover, the transmission of the parasites between livestock and humans may be facilitated through contaminated water.

We investigated the prevalence of Cryptosporidium oocyst and Giardia cyst in water used in dairy farms as well as the tap water in urban areas in Taiwan.

Water samples were collected between April to August 2004, at the areas shown by filled circle and the number of collected samples shown in parenthesis in Fig. 1. A total of twenty-five tap water samples from school, recreational park, household and temple were collected (Table 1). Another thirty water samples were collected from water faucets at livestock farms in Taichung county and in Tainan. Of these, sixteen water samples were from 5 cattle farms, six from 3 farms that kept both goats and cattle, three from 1 goat farm and five from 1 farm that raises various kinds of animals including horses. When the water sample was collected, we also enquired about the water sources. If it is an underground water, the depth of the underground well, as well as, if it is being used for livestock drinking water or not.

Water sample collection, parasite isolation and detection were conducted as described by the Japan Water Works Association (JWWA) [8]. This method is considered to be equivalent to method 1622 as designated by the United States Environmental Protection Agency (EPA) [18]. Briefly, 201 of water from the faucet were filtered through a $5.0 \mu \mathrm{m}$ pore size PTFE membrane filter (Millipore, Japan) placed in a filtration apparatus (Advantec, Japan). Protozoa trapped on the filter membrane were eluted using PET eluting solution by vortexing [7]. The eluate was then concentrated by centrifugation in a $50 \mathrm{~m} l$ centrifuge tube. Protozoa were isolated using the immunomagnetic separation system (IMS) as described by JWWA. The sample suspension were immunostained, follow by microscopic examination. 


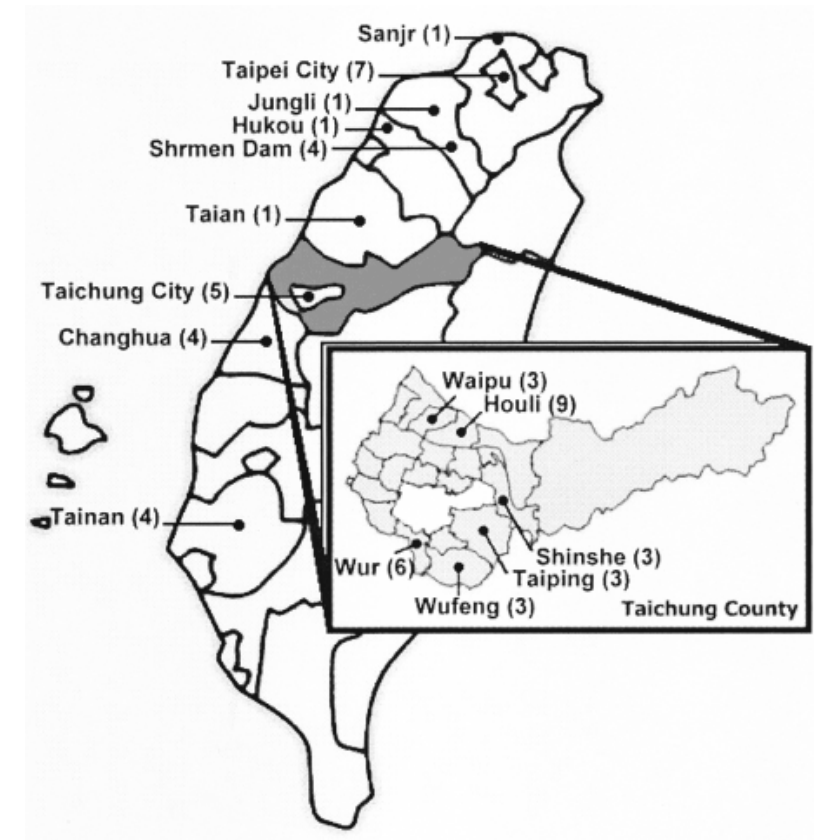

Fig. 1. Locality (filled circle) and number of water samples (in parenthesis) collected for examination of Cryptosporidium oocyst and Giardia cyst.

Table 1. Feature of sampling site and the number of samples collected

\begin{tabular}{llc}
\hline Sampling site & Sample type & No.examined \\
\hline City tap water & School & 10 \\
& Recreational park & 10 \\
& Household & 4 \\
& Temple & 1 \\
\hline Farm area & Underground water* & \\
& 5 & 3 \\
& 20 & 4 \\
40 & 3 \\
& 80 & 3 \\
& 396 & 4 \\
& 429 & 2 \\
& unknown & 1 \\
& Mountain spring & 4 \\
& River water & 1 \\
& Tap water & 5 \\
\hline Total & & 55 \\
\hline
\end{tabular}

*: Depth in meters.

Concentrated water samples sediments were then stained with FITC-conjugated anti-Cryptosporidium and anti-Giardia monoclonal antibody (Biotechnology Frontier, Australia) and with DAPI (4'-6'-diamidino-2-phenylindole). The samples were then observed under ultraviolet light fluorescence microscope at $400 \times$ magnification (Olympus, Japan). Oocysts and cysts were identified based on the following parameters; size, shape, surface feature and staining reaction. Particles exhibiting the right size and shape as that of the protozoa were further identified according to their internal structure, especially their nuclei, when stained by DAPI. Whole microscopic fields were observed and all the parasites were counted.

One $(1.82 \%)$ out of 55 water samples was positive for Cryptosporidium oocyst and 2 (3.64\%) out of 55 samples were positive for both of Cryptosporidium oocyst and Giardia cyst (Table 2$)$. Only 1 oocyst $(0.05$ oocyst/l) was obtained in the Cryptosporidium-positive, Giardia-negative water sample. In the two other samples that were both Cryptosporidium and Giardia positive, 1 oocyst $(0.05$ oocyst $/ l)$ and 2 cysts $(0.1$ cysts $/ l)$ were observed in one sample, and 2 oocysts $(0.1$ oocyst $/ l)$ and 1 cyst $(0.05 \mathrm{cyst} / l)$ were observed in the other sample, respectively. No Cryptosporidium oocyst or Giardia cyst was detected in the 25 city tap water samples tested. In one underground-water sample that was positive for Cryptosporidium oocyst, the same water source was used as drinking water for goats in that farm. Two water samples from mountain spring that were positive for both of Cryptosporidium oocysts and Giardia cysts, were also used as a source of drinking water for both cattle and goats. Since the number of oocysts or cysts detected was very low, no attempt was made to carry out the genotyping of the oocysts.

High prevalence of Cryptosporidium spp. infection in cattle has been reported in many countries [2, 16]. Cryptosporidium parvum has recently been implicated in waterborne outbreaks in developed $[10,14]$ and developing countries [17]. C. parvum oocysts had been reported in river water in dairy farming area of Hokkaido, Japan [15]. In Taiwan, Cryptosporidium and Giardia were reportedly found in raw water samples from river water $[5,6]$. This indicates the potential contamination of drinking water and can threaten the health of people living in both urban and rural area in Taiwan proper.

Significant restructuring of normal dairy farm to one that cater for recreational purpose in Taiwan was evident in that, in 2000 , there were only 22 leisure farms, but increased to over 200 in 2004 . Thus, the chance of the public having direct contact with farm animals or the environment that might be contaminated with Cryptosporidium oocyst or Giardia cyst excreted from farm animals, had increased.

In Taiwan, little has been done to check the tap water and the drinking water for farm animals for waterborne protozoa. In our study, we found Cryptosporidium oocysts and Giardia cysts in 3 water samples from the dairy farms, but none in the city tap water. It might be possible that the source of the contamination might come from animals that had been infected with both parasites. Since the city tap water were negative for the target waterborne protozoa, it indicates the good sanitary condition of the city tap water in Taiwan. However, there is still a potential danger for zoonotic transmission of the protozoan to human because same source of water is used as drinking water for both animals and humans. Underground water, mountain spring, river water and treated tap water were used as drinking water for livestock in Taiwan. The depth of the 
Table 2. Prevalence of Cryptosporidium oocysts and Giardia cysts in various water samples in Taiwan

\begin{tabular}{lcccc}
\hline & No. examined & \multicolumn{2}{c}{ No.positive } \\
& & $\begin{array}{c}\text { Cryptosporidium } \\
\text { only }\end{array}$ & Giardia only & $\begin{array}{c}\text { Cryptosporidium } \\
+ \text { Giardia }\end{array}$ \\
\cline { 2 - 5 } Tap water in urban area & 25 & 0 & 0 & 0 \\
Farm area & 21 & 1 & 0 & 0 \\
$\quad$ Underground water & 4 & 0 & 0 & 2 \\
$\quad$ Spring & 5 & 0 & 0 & 0 \\
$\quad$ Tap water & 55 & $1(1.82 \%)$ & 0 & $2(3.64 \%)$ \\
\hline Total & & &
\end{tabular}

underground well ranged from $20-429 \mathrm{~m}$. In the three farms positive for Cryptosporidium oocyst, 2 used mountain spring water as drinking water for the animals. Since mountain water are easily exposed to livestock than underground water, the former might be more prone to contamination by the protozoa than the latter. Viability of C. parvum oocyst in the soil has been reportedly to be more than 120 days in an experiment by Kato et al. [9]. It has been proposed that an outbreak of human cryptosporidiosis would probably occur if the tap water contained more than 10 Cryptosporidium oocysts/100l [3]. In our study, 2 oocysts/20l were found in a water sample derived from one dairy farm. This indicates the potential risk of a waterborne outbreak should not be ignored. We recommend that surveillance of parasite contamination of water in dairy farms, including those of the leisure farms should be carried out regularly to ensure the safety and hygienic quality of the water. This is the first report of the finding of Cryptosporidium oocyst and Giardia cyst in the drinking water of livestock.

ACKNOWLEDGMENTS. This study was funded by a grant-in-aid from the Bureau of Animal and Plant Health Inspection and Quarantine, Council of Agriculture, Executive Yuan, Taiwan (Grant no. 94AS-13.3.1-BQ-B3). We thank Mr. Hsu, H. C. and Mr. Ho, S. Y. for the assistance in sampling and shipping of water samples.

\section{REFERENCES}

1. Current, W. L., Reese, N. C., Ernest, J. V., Bailey, W. S., Heyman, M. B. and Weinstein, W. M. 1983. New Engl. J. Med. 308: $1252-1255$.

2. Fayer, R. 2004. Vet. Parasitol. 126: 37-56.
3. Haas, C. N. and Rose, J. B. 1995. J. Am. Water Works Assoc. 88: $81-84$.

4. Hashimoto, A., Kunikane, S. and Hirata, T. 2002. Water Res. 36: 519-526.

5. Hsu, B. M., Huang, C. P., Lilian Hsu, C. L., Hsu, Y. F. and Yeh, J. H. 1999. Water Res. 33: 2701-2707.

6. Hu, T. L. 2002. J. Microbiol. Immunol. Infect. 35: 68-70.

7. Inoue, M., Rai, S. K., Oda, T., Kimura, K., Nakanishi, M., Hotta, H. and Uga, S. 2003. J. Microbiol. Methods 55: 679686.

8. Japan Water Works Association (JWWA) 2003. pp. 50-55 JWWA. (in Japanese).

9. Kato, S., Jenkins, M., Fogarty, E. and Bowman, D. 2004. Sci. Total Environ. 321: 47-58.

10. Kuroki, T., Watanabe, Y., Asai, Y. and Yamai, S. 1996. J. Jpn. Assoc. Inf. Dis. 70: 132-140.

11. Lindsay, D. S., Upton, S. J., Owens, D. S., Morgan, U. M., Mead, J. R. and Blagburn, B. L. 2000. J. Eukaryot. Microbiol. 47: 91-95.

12. Lu, W. J. and Kao, S. J. 1998. Anim. Health Bull. 8: 1-2.

13. Robertson, L. J. and Gjerde, B. 2001. Scand. J. Public Health 29: 200-207.

14. Smith, H.V. and Rose, J. B. 1998. Parasitol. Today 14: 14-22.

15. Tsushima, Y., Karanis, P., Kamada, T., Nagasawa, H., Xuan, X., Igarashi, I., Fujisaki, K., Takahashi, E. and Mikami, T. 2001. J. Vet. Med. Sci. 63: 233-236.

16. Uga, S., Matsuo, J., Kono, E., Kimura, K., Inoue, M., Rai, S. K. and Ono, K. 2000. Vet. Parasitol. 94: 27-32.

17. Uga, S., Oda, T., Kimura, K., Kimura, D., Setiawan, K., Sri, M., Nuvit, K. and Apakupakul, N. 2003. Jpn. J. Trop. Med. Hyg. 31: 87-91.

18. United States Environmental Protection Agency. 1999. Method 1622. Cryptosporidium in Water by Filtration/IMS/FA. U. S. EPA Office of Water.

19. Watanabe, Y., Yang, C. H. and Ooi, H. K. 2005. Parasitol. Res. 96 (in press) 